\title{
EMERGENCIA Y DESARROLLO DE LAS \\ HABILIDADES METALINGÜÍSTICAS \\ RELACIONADAS CON LA REGULACIÓN DE LA INFORMACIÓN: UN ESTUDIO TRANSVERSAL EN NIÑOS DE EDADES COMPRENDIDAS ENTRE LOS 22 MESES Y LOS 10 AÑOS
}

\author{
EMERGENCE AND DEVELOPMENT OF INFORMATION- \\ REGULATION METALINGUISTIC ABILITIES: A CROSS-SECTIONAL \\ STUDY IN CHILDREN AGED 22 MONTHS TO 10 YEARS
}

\author{
Ma ISABEL NAVARRO RUIZ \\ Universidad Autónoma de Barcelona, España. \\ MariaIsabel.Navarro@uab.cat
}

CONCHI SAN MARTÍN

Universidad de Barcelona, España.

csan_martin@uoc.edu

\section{RESUMEN}

El objetivo de este trabajo es observar la emergencia y desarrollo de las habilidades metalingüísticas relacionadas con la regulación de la información mediante un estudio transversal. Intervinieron 40 participantes de edades comprendidas entre los 22 meses y los 10 años. La lengua materna y la usada en la adquisición de conocimientos eran el catalán y el castellano. Se utilizó una metodología observacional en un contexto comunicativo natural y espontáneo entre el niño y el adulto. Se elaboró un sistema de categorías (Navarro y Hernández, 2004; Navarro, 2007; Navarro y San Martín, 2007) dividido en dos grupos: Regulación y ajustes de la comunicación y Regulación basada en el interlocutor como centro de interés. Los resultados nos indican que el grupo categorial Regulación y ajustes de la comunicación aparece a una edad muy temprana y se mantiene activo prácticamente a lo largo de todas las edades observadas. Inicialmente predominan aquellas categorías encaminadas a la obtención de la información, a la vez que el niño también la facilita para poder mantener una interacción de forma adecuada. Respecto al grupo de categorías Regulación basada en el interlocutor como centro de interés aparece tardíamente, cuando el niño posee un mayor grado de competencia, excepto la categoría Referencia al habla o al idioma de los demás que se ha detectado a una edad más temprana.

Palabras claves: Metapragmática, regulación de la información, metalingüística, desarrollo de las habilidades metalingüísticas. 


\begin{abstract}
The aim of this work is to observe the emergence and development of metapragmatic skills related to the regulation of information. A transversal study was carried out, made up of 40 participants, aged between 22 months and 10 years. The mother tongue and that used for acquisition of knowledge were Catalan and Spanish. An observational methodology was used, recording interactive situations between the child and the adult. A category system was developed, divided into two groups: Regulation and adjustments of communication and Regulation based on the interlocutor as the centre of interest. The results show us that the Regulation and adjustments of communication category group appear at a very early age and is maintained active practically throughout all the ages observed. Initially the categories aimed at the obtaining of information predominate, while the child also facilitates information in order to maintain adequate interaction. Regarding the group of Regulation categories based on the interlocutor as the centre of interest, this appears at a later stage, when the child possesses a greater degree of competence, except the category Reference to the speech or language of others, which has been detected at an earlier age.
\end{abstract}

Keywords: Metapragmatics, regulation of information, metalinguistics, development of metalinguistic skills.

Recibido: 19-06-2008. Aceptado: 28-11-2008.

\title{
1. INTRODUCCIÓN
}

G $\mathrm{n}$ los intercambios comunicativos que realizamos en nuestra vida cotidiana, Cse producen desajustes que una vez detectados suelen solventarse a través de estrategias verbales diversas. En este tipo de actividades podemos encontrar producciones en las que el lenguaje se convierte en el objeto de atención de los interlocutores, registrándose intercambios de carácter metalingüístico que permiten en numerosos casos que la información se regule y gestione con éxito, por ejemplo cuando los interlocutores facilitan información sobre diferentes aspectos del mensaje o cuando formulan peticiones de información. Con ello se resuelven ambigüedades lingüísticas, se ayuda a la comprensión de los mensajes y se negocian los acuerdos necesarios para una correcta interacción.

En el caso de la evolución de las habilidades metalingüísticas en edades infantiles, encontramos que el estudio de éstas ofrece una gran diversidad de resultados, cuestión que en ocasiones ha dificultado trazar un panorama claro de su desarrollo. Sin embargo, en la actualidad habría un cierto consenso en considerar que si bien son procesos de adquisición lenta que se consolidan tardíamente también es posible detectarlos, aunque de un modo oscilante y embrionario, en edades tempranas. En este sentido Gombert (1990) afirma que inicialmente emergen las denominadas formas epilingüísticas, provistas de una aparente coincidencia formal con los comportamientos metalingüísticos, pero desprovistas de un carácter 
consciente. No será hasta los 6 ó 7 años, según el citado autor, cuando realmente aparezcan los comportamientos de carácter metalingüístico, es decir, comportamientos provistos de reflexión consciente.

Según lo expuesto, los objetivos del presente trabajo se centran en dos cuestiones:

1. Profundizar en las secuencias de desarrollo y estabilización de las habilidades metalingüísticas, así como en los momentos más o menos oscilantes y embrionarios de la emergencia de éstas. Para ello resulta necesario trabajar con amplias franjas de edad que permitan indicar los logros y dificultades infantiles en el acceso a las habilidades metalingüísticas relacionadas con la regulación de la información.

2. Analizar estas cuestiones en un contexto comunicativo natural y espontáneo donde pueda contextualizarse la emergencia de estas habilidades respecto a las interacciones habidas entre un interlocutor más experto (en este caso un adulto) y el niño.

\section{MARCO TEÓRICO}

En la literatura pueden apreciarse diferentes definiciones sobre la función metalingüística, pero en general ésta se puede considerar como: "la capacidad de distanciarse del uso normal del lenguaje y de apartar la atención de los objetivos de la comunicación para dirigirla hacia las propiedades del lenguaje utilizado como medio de comunicación" (Rondal, Espert, Gombert, Thibaut \& Comblain, 2003: 42). La función metalingüística contemplada desde este punto de vista trata de la reflexión sobre el lenguaje y sus reglas, y sobre el control del proceso de producción y comprensión del lenguaje (Gombert, 1990).

Respecto a la evolución de las habilidades metalingüísticas, existe un acuerdo general en remarcar la influencia decisiva de éstas en el desarrollo del lenguaje, actuando la interacción entre ambas habilidades como un sistema de retroalimentación. Según Karmiloff-Smith (1986), este avance se consigue gracias a la redescripción sucesiva de nuevos conocimientos. En este progreso el niño debe pasar a través de distintas fases, comenzando por el conocimiento implícito acerca del lenguaje hasta llegar al conocimiento explicitado al final de este proceso, momento en el cual el niño será capaz de verbalizar sus reflexiones sobre el lenguaje.

Por otro lado, el concepto habilidades metalingüísticas engloba diferentes niveles de lenguaje, como es el caso de la habilidad metapragmática. Ésta implica una reflexión sobre la relación existente entre el sistema lingüístico y el contexto en que es usado, así como una toma de conciencia de los procesos que rigen la comunicación. Según Hickman (1985), se trata de la habilidad que permite representar, 
organizar y regular el uso de la propia habla.

El desarrollo de estos procesos puede rastrearse en los primeros actos de comunicación preverbal, basados en la acción y en la atención conjunta donde el adulto juega un papel importantísimo, al principio dirigiéndose al niño como si pudiera comprender cuanto está diciendo, atribuyendo un supuesto significado a las actuaciones del pequeño que a su vez podrá ir captando progresivamente la intención comunicativa del adulto, así como el significado que éste confiere a cada gesto, sonido, etc. Es en estas primeras interacciones donde se asienta la futura base del desarrollo lingüístico y metalingüístico.

Más tarde, en el período lingüístico los interlocutores podrán mantener una conversación, pero para que ésta tenga éxito los participantes deberán ser capaces de gestionar la información de que disponen (dando o pidiendo, por ejemplo, aquella que crean conveniente para mantener la interacción). En el desarrollo de esta capacidad se pueden observar claros movimientos evolutivos, así a los 24 meses las peticiones contingentes de información tienen mayoritariamente un carácter no verbal; a los 30 meses los niños responden a las preguntas aunque sin demasiada eficacia, pero si la petición de información se refiere a sus propias acciones mejora su actuación (Anderson, 1992). No será hasta aproximadamente los 3 años cuando los niños muestren un conocimiento más amplio sobre la semántica y la pragmática del discurso, así como sobre las estrategias de regulación de la comunicación que conlleva el diálogo. A los 4 años los niños presentan aún grandes limitaciones para responder correctamente a las preguntas formuladas por su interlocutor, siendo hacia los 5 años cuando se aprecia una notable evolución en este aspecto, mostrando el pequeño más autonomía discursiva y habilidad a la hora de facilitar o demandar información (Karmiloff \& Karmiloff-Smith, 2001).

Otro aspecto íntimamente relacionado con el establecimiento y mantenimiento de un diálogo fluido y eficaz es que el hablante comprenda la importancia de conocer la cantidad y calidad de información que posee su interlocutor, así como la información que se le debe proporcionar (Beaudichon \& Ducroux, 1985). Ello supone que los hablantes han de ser capaces de ponerse en el lugar del otro y adecuarse a su conocimiento. Según Anderson (1992), al principio el niño no comprende el efecto que tiene sobre el oyente el hecho de no proporcionar suficiente información. Esta actitud mejora notablemente a partir de los 3 años, aunque todavía los pequeños tienden a pensar que su interlocutor dispone de todos los conocimientos necesarios para poder comprenderle, apareciendo consecuentemente limitaciones y dificultades a la hora de sopesar la información que debe facilitar. Evidencian este hecho las conversaciones telefónicas que podemos mantener con un pequeño de esta edad (el niño nos habla de referentes que tiene ante sí e incluso los señala empleando deícticos, pero sin entender que su interlocutor no dispone de la información referencial pertinente). Siguiendo esta línea de trabajo, Clark (1978) pudo comprobar que de los 3 a los 4 años los niños pueden detectar si el 
oyente ha comprendido o no su emisión y, en caso negativo, son capaces de facilitar información y de repetir su producción con algunas variantes.

Por otra parte, trabajos clásicos como los de Markman (1977, 1979, 1981) muestran también las dificultades de los pequeños en el manejo de las ambigüedades informativas. El citado autor realizó distintos estudios en los que se proporcionaban mensajes que contenían diversas inconsistencias o contradicciones, comprobando que los niños de 5 años no las detectaban. También pudo verificar que detectar inconsistencias en un mensaje es un proceso arduo para el pequeño, no pudiendo realizar correctamente las tareas propuestas hasta los 11 años. No obstante, los niños obtienen mejores resultados si la inconsistencia está vinculada a su experiencia, en estos casos los pequeños de 5 años mejoran su actuación en este tipo de tareas (Tunmer, Bowey \& Grieve, 1983).

\section{METODOLOGÍA}

Se realizó un estudio trasversal de las habilidades metalingüísticas relacionadas con la regulación de la información.

\subsection{Participantes}

La muestra está compuesta por 40 participantes, éstos son niños residentes en Cataluña que reunían las siguientes características:

Edad: Según los datos extraídos de la literatura consultada, podemos observar que las primeras emergencias epilingüísticas suelen aparecer alrededor de los 2 años de edad y hacia los 10 años presentan unas habilidades metalingüísticas notablemente evolucionadas, por esta razón la muestra está comprendida entre los 22 meses $\mathrm{y} \operatorname{los} 10$ años.

Género: Con el fin de evitar variables extrañas asociadas al género se seleccionó para cada edad observada un niño y una niña, quedando la muestra constituida por 20 niñas y 20 niños.

Variables sociodemográficas: Dado que la variable nivel sociocultural influye en el desarrollo de las habilidades metalingüísticas, nos proponemos controlar ésta mediante la selección de niños procedentes de familias con nivel sociocultural medio.

Lengua: Los niños participantes provienen de un contexto bilingüe (castellano/catalán), por lo que se decidió seleccionar aquellos que estuvieran expuestos a ambas 
RLA. Revista de Lingüística Teórica y Aplicada, 47 (1), I Sem. 2009

lenguas, tanto en el contexto familiar como en el educativo.

\subsection{Procedimiento}

Se emplea un diseño observacional. En ningún momento se provocó la aparición de las habilidades metalingüísticas con el fin de salvaguardar la validez ecológica del trabajo, mostrando de este modo la frecuencia de uso normal de estas producciones en una situación de interacción, razón por la que el entrevistador actuaba como participante externo en una situación natural. Ésta fue la misma persona a través de todo el estudio, controlándose así la variable estilo discursivo del interlocutor.

Se estableció un total de 20 momentos evolutivos para su observación, iniciándose ésta a los 22 meses. Los intervalos entre observaciones fueron de 4 meses para las edades comprendidas entre los 22 meses y los $4 a .4 \mathrm{~m}$. A partir de esta edad, las observaciones se realizaron en intervalos de 6 meses. Se optó por este ritmo de observación debido a que antes de los 4 años se produce un cambio mucho más rápido de las competencias lingüísticas. Para cada momento evolutivo se observaron dos participantes, una niña y un niño, por lo que se obtuvo un total de 40 observaciones.

Las diferentes producciones fueron registradas en cintas de audio/vídeo. Posteriormente fueron transcritas, utilizando el sistema de transcripción Systematic Analysis of Language Transcript (SALT) (Miler \& Chapman, 1985). Se tomó como unidad de análisis la oración y se optó por el criterio gramatical para su identificación. En caso de ambigüedad se siguió el criterio entonacional como delimitación de la oración. Se siguen, por tanto, las directrices de Crystal, Fletcher $\&$ Garman (1976), ya que este criterio nos es útil para el estudio de participantes que siguen la pauta estándar de desarrollo y niños que presentan un déficit de lenguaje (Navarro y Hernández, 2004, Navarro, 2007). En cada registro se rechazaron los primeros 5 minutos con el fin de eliminar producciones que pudieran ser fruto del nerviosismo propio del momento o cualquier otro elemento distractor que se hubiera podido producir al inicio de la grabación, a continuación se contabilizaron las primeras 100 oraciones para su estudio.

\subsubsection{Materiales}

Los objetos usados en los diferentes registros los aportaba la entrevistadora en un gran saco que contenía juguetes (pelotas, muñecos de diversos tipos, coches), otros materiales figurativos y libros de cuentos con grandes ilustraciones. Estos fueron usados en el transcurso de cada entrevista según los intereses y necesidades del niño. 


\subsubsection{Entrevista}

Siguiendo el criterio de Berthoud-Papandropoulou (2000), se ha buscado una situación funcional para realizar este trabajo, ya que según la citada autora estas situaciones permiten al niño mostrar de modo espontáneo su conocimiento metalingüístico. Es por ello que se optó por una entrevista semiestructurada.

Para las entrevistas se utilizó habitualmente una sala en la escuela, espaciosa y con la posibilidad de sentarse y jugar en el suelo, así como una mesa con sillas a la medida del niño. En la sala sólo estaban presentes la entrevistadora y el pequeño.

Las entrevistas tuvieron una duración aproximada de 45 minutos. En ellas el participante y la entrevistadora jugaban y hablaban sobre temas comunes para todos los niños que fueron entrevistados, siendo éstos de interés para el pequeño y próximos a su entorno. Se mantuvo un contacto previo para evitar el efecto de reactancia que pudiera presentar el niño ante una persona desconocida.

\subsubsection{Sistema de categorización}

Las categorías de regulación de la comunicación utilizadas en este trabajo provienen de un sistema de categorización más extenso (Navarro y Hernández, 2004), elaborado para comparar la influencia de distintos escenarios y también útil a la hora de comparar distintos comportamientos metalingüísticos que podrían estar asociados a las patologías del lenguaje (Navarro, 2007; Navarro y San Martín, 2007).

Las categorías que presentamos a continuación han sido extraídas del sistema anteriormente mencionado con el objetivo de estudiar un aspecto concreto del comportamiento metalingüístico como es la regulación de la información. Están divididas en dos grupos: a) Regulación y ajustes de la comunicación y b) Regulación basada en el interlocutor como centro de interés. Dentro del primer grupo se contemplan cuatro categorías: Dar información de carácter metalingüístico, Obtener información de carácter metalingüistico, Detección de incoherencias en el discurso y Reorganización de la información. Dentro del segundo grupo se contemplan tres categorías: Referencia a lo dicho por el interlocutor, Adecuación discursiva y Referencia a las caracteristicas del habla o idioma de los interlocutores. La definición operativa de las mismas y los ejemplos de aplicación pueden consultarse en el Anexo 1.

Se procedió, por último, a comprobar la fiabilidad de las categorías mediante un análisis interjueces, obteniendo una concordancia interobservadores entre $k=.85$ y .87 . 


\section{RESULTADOS}

Se muestra, en primer lugar, la evolución de las categorías en función de la edad, considerándose las frecuencias de uso, el momento evolutivo donde se detecta la primera aparición de cada categoría y las correlaciones con la edad. En segundo lugar, se analiza de manera cualitativa la emergencia de las categorías.

\subsection{Evolución de las categorías en función de la edad}

Se ha obtenido un total de 291 unidades categorizadas en los 20 puntos evolutivos de observación. Las frecuencias y porcentajes totales, independientemente de la edad, quedan recogidos según los grupos categoriales: Regulación y ajustes de la comunicación (ver Tabla I) y Regulación basada en el interlocutor como centro de interés (ver Tabla II). Las frecuencias de uso de las categorías en función de la edad quedan recogidas también en función de los grupos categoriales (ver gráficos 1 y 2 , respectivamente).

Los resultados nos indican que el grupo categorial Regulación y ajustes de la comunicación aparece a una edad muy temprana ( 22 meses) y se mantiene activo prácticamente a lo largo de todas las edades observadas, aunque con diferentes oscilaciones e intensidades. Las categorías más frecuentes en este grupo son Obtener información $(41,99 \%)$ de especial uso en las primeras edades observadas, seguida de Dar información (35,36\%), mientras que la categoría menos frecuente es la de Determinar incoherencias $(7,18 \%)$.

Tabla I. Porcentajes de las categorías pertenecientes al grupo 1:

Regulación y ajustes de la comunicación.

\begin{tabular}{|c|c|}
\hline Categorías & Porcentaje (frecuencia) \\
\hline Dar información de carácter metalingüístico & $35,36 \%(64)$ \\
\hline Obtener información de carácter metalingüístico & $41,99 \%(76)$ \\
\hline Detección de incoherencias en el discurso & $7,18 \%(13)$ \\
\hline Reorganización de la información & $15,47 \%(28)$ \\
\hline Total & $100,00 \%(181)$ \\
\hline
\end{tabular}




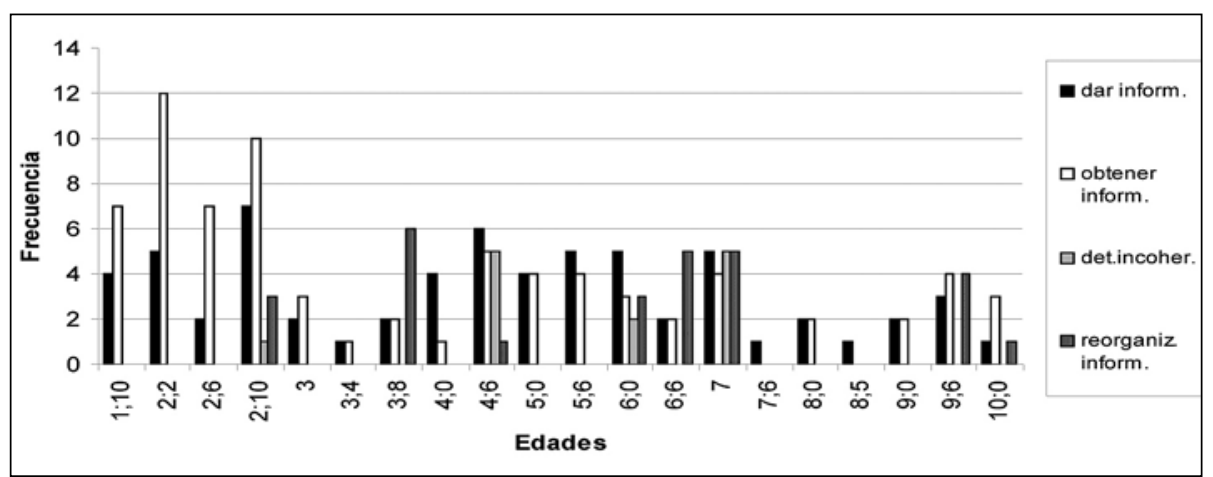

Gráfico 1. Frecuencias del grupo categorial 1: Regulación y ajustes de la comunicación: evolución en función de la edad.

En el grupo categorial Regulación basada en el interlocutor como centro de interés la categoría más frecuente es Referencia a lo dicho por el interlocutor (55,45\%), que además muestra un perfil de evolución más activo entre los 4;6 y 8 años. La categoría Referencia al habla muestra menor frecuencia (30,91\%), mientras que la categoría restante, Adecuación discursiva, presenta una escasa frecuencia de uso en el conjunto de la muestra $(13,64 \%)$.

Tabla II. Porcentajes de las categorías pertenecientes al grupo 2:

Regulación basada en el interlocutor como centro de interés.

\begin{tabular}{|c|c|}
\hline Categorías & Porcentaje (frecuencia) \\
\hline Referencia a lo dicho por el interlocutor & $55,45 \%(61)$ \\
\hline Adecuación discursiva & $13,64 \%(15)$ \\
\hline $\begin{array}{l}\text { Referencia a las características del habla o idioma } \\
\text { de los interlocutores }\end{array}$ & $30,91 \%(34)$ \\
\hline Total & $100,00 \%(110)$ \\
\hline
\end{tabular}




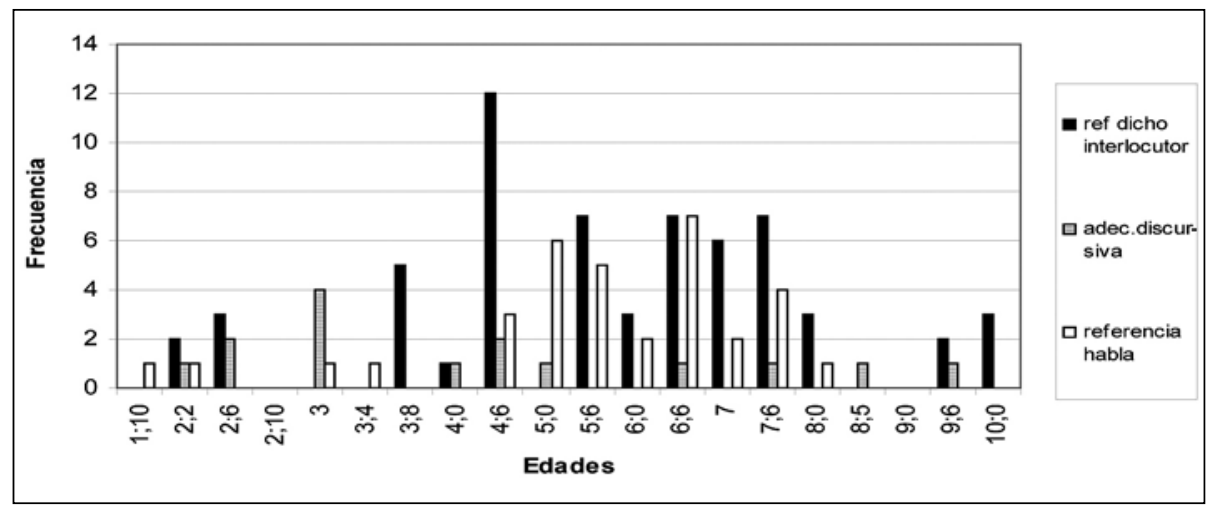

Gráfico 2. Frecuencias del grupo categorial 2: Regulación basada en el interlocutor como centro de interés: evolución en función de la edad.

Para poder analizar la relación existente entre la frecuencia de las diferentes categorías y la edad -expresada en meses- en que aparecen, se empleó el índice de correlación de Pearson, obteniéndose una correlación negativa para las categorías: Dar información y Obtener información con una significación de $p<.05$.

$\mathrm{Si}$ consideramos estos resultados conjuntamente con la edad de emergencia de cada categoría, tal y como queda recogido en la Tabla 3 en orden cronológico, observamos que las categorías Obtener información y Dar información aparecen de manera temprana y disminuyen significativamente con la edad, mientras que la categoría Referencia al habla también aparece de manera temprana, pero sin que se observen variaciones significativas en función de la edad. El resto de categorías son de aparición más tardía y en ninguno de los casos se observa una variación significativa en función de la edad.

Tabla III. Edad de emergencia de las categorías.

\begin{tabular}{lc}
\hline \multicolumn{1}{c}{ Categoría } & Edad de detección \\
Dar información (grupo categorial 1) & $1 ; 10$ \\
Obtener información (grupo categorial 1) & $1 ; 10$ \\
Referencia habla (grupo categorial 2) & $1 ; 10$ \\
Referencia a lo dicho (grupo categorial 2) & 2,2 \\
Adecuación discursiva (grupo categorial 2) & 2,2 \\
Detección de incoherencias (grupo categorial 1) & $2 ; 10$ \\
Reorganización de la información (grupo categorial 1) & $2 ; 10$ \\
\hline
\end{tabular}

Grupo categorial 1: Regulación y ajustes de la comunicación

Grupo categorial 2: Regulación basada en el interlocutor como centro de interés. 


\subsection{Análisis cualitativo de la emergencia y evolución de las categorías}

A continuación se presenta un análisis cualitativo para contextualizar la emergencia y evolución de cada una de las categorías estudiadas. En este análisis se integrarán en parte los resultados obtenidos en el análisis cuantitativo y se realiza también una lectura interpretativa de los mismos.

\section{Obtener información de carácter metalingüistico}

En el análisis cuantitativo se ha observado que inicialmente predominan aquellas categorías encaminadas a la obtención de la información, este hecho puede interpretarse entendiendo que en estas primeras edades el niño necesita mayor información de su interlocutor por estar en la fase de los inicios del desarrollo del lenguaje. Sin embargo, con la edad se detecta una modificación en el tipo de información requerida, así a los $3 \mathrm{a} .4 \mathrm{~m}$. puede apreciarse el deseo del niño de obtener información mucho más precisa, apareciendo de forma explícita preguntas sobre el significado tal y como se recoge en el siguiente ejemplo:

Niño

$$
\begin{aligned}
& \text { : ¿rápido es esto? } \\
& \text { \{Echa a correr }
\end{aligned}
$$

Este comportamiento coincide con edades en las que el niño está adquiriendo nuevos conceptos, a la vez que desarrolla las relaciones que se establecen en el triángulo semiótico. En el mismo sentido aparecen sobreextensiones que suponen un problema para el pequeño que debe resolver.

Niña

$$
\text { : ¿El cielo es el techo de la calle? }
$$

\section{Dar información de carácter metalingüistico}

Según se ha indicado en el análisis cuantitativo, el pequeño también es capaz de Dar información precozmente si detecta o piensa que su interlocutor la necesita para poder comprender el mensaje que está emitiendo. Estas cuestiones también muestran importantes variaciones a nivel cualitativo. Al principio el niño parece dar por supuesto en muchos casos que el interlocutor tiene la misma información que él y, por tanto, no la facilita. Más tarde se detecta en algunas ocasiones un exceso de información debido a una falta de dominio de las máximas de cortesía. Con el tiempo el pequeño gana en precisión y competencia a la hora de ponerse en lugar de su interlocutor y determinar la información que debe facilitar. En los siguientes ejemplos observamos la ampliación de información realizada por una niña de 22 meses al pensar que el adulto necesita más datos para poder entenderla: 
\{La niña enseña a la entrevistadora el atuendo que viste. El adulto le pregunta por las bambas\}

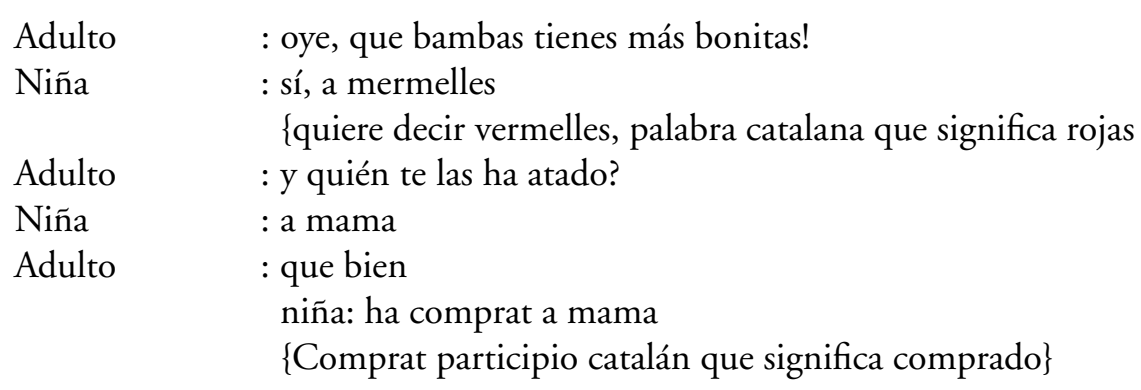

$\{$ La niña y la entrevistadora se encuentran en la clase jugando. A la hora del patio los niños se bañan en una piscina, por esta razón han de llevar un bañador y unas zapatillas de goma. El colegio dispone también de zapatillas de goma en el caso de que algún niño las olvide\}.

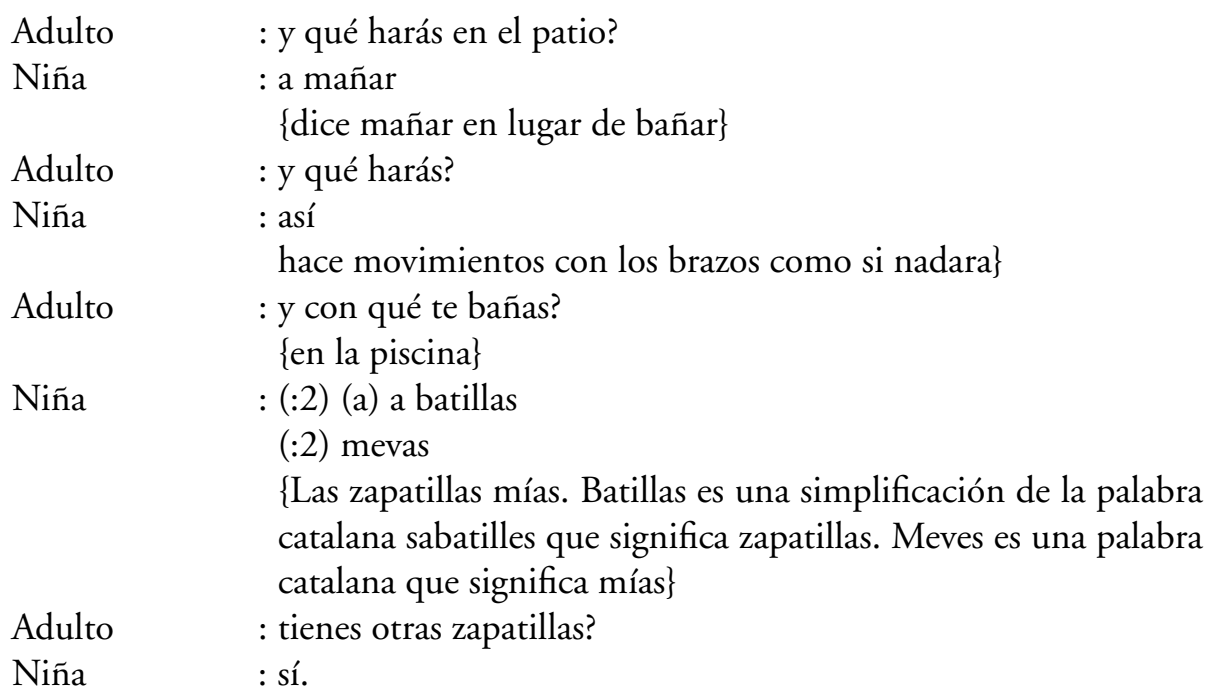

En la franja de edad comprendida entre los 4a.6m y los 5a.6m. de edad se ha observado a nivel cuantitativo un repunte en la frecuencia de uso de esta categoría. En esta franja de edad se han detectado producciones como la siguiente:

\{Niña de 5a.6m. Habla de un regalo que le ha hecho el Ratoncito Pérez. La niña facilita más información para que el interlocutor no incurra en una confusión respecto a un conocimiento socialmente compartido\} 
Niña

: Ha venido el Ratoncito Pérez (:2)

¡Pero no se me ha caído un diente!

\{Habitualmente el ratoncito Pérez visita a los niños cuando se les ha caído un diente se lleva éste y a cambio les deja un regalo, pero en esta ocasión visita a la niña sin que ésta haya perdido ninguna pieza de su dentadura\}

Entre los 5a.6m. y 7 años aparece nuevamente esta categoría cobrando un carácter distinto. Ahora en la mayoría de estas producciones el niño se pone en lugar del adulto y piensa que tal vez éste necesita más información para poder proseguir con éxito la conversación, como se aprecia en el ejemplo siguiente correspondiente a un niño de 6 años:

Niño

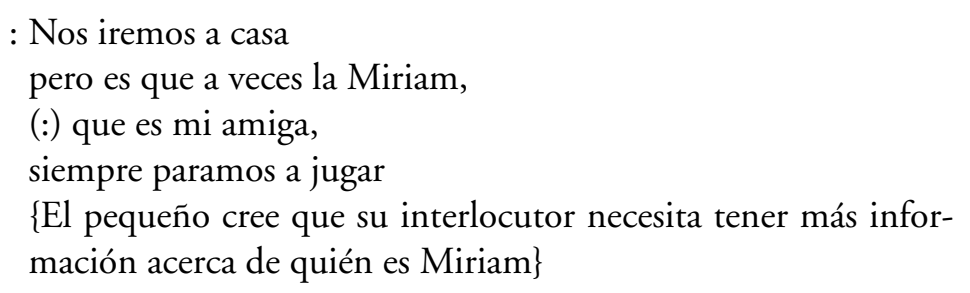

En el siguiente ejemplo el entrevistador sólo pregunta al niño dónde irá de vacaciones. El pequeño facilita la información que cree necesaria y la amplía, aunque en exceso.

\{Niño 7 años. Habla de sus próximas vacaciones\}

Adulto $\quad: ¿$ Dónde irás de vacaciones?

Niño

: (y y que) y me lleva a una piscina

si cada día cuando tengo (:) una (:)

cuando $\mathrm{ca}^{*}$ ) cuando tenia cinco años fui

cuando tenía seis años fui

(cuando tenía) ahora como tengo siete años voy a ir

y (hasta cuando) hasta cuando tenga diez

si (es) (el dia) cuando cumpla los once ya no voy

porque ya soy mayorcito y $\left(\mathrm{se}^{*}\right)$ ya sabré nadar

porque (me a) a mí me gusta meterme por el fondo del agua

(pero ya no) pero ahora me van a comprar las gafas de piscina 
RLA. Revista de Lingüística Teórica y Aplicada, 47 (1), I Sem. 2009

\section{Detección de incoherencias en el discurso}

Destacamos el contenido de esta categoría cuando se focaliza en pedir información con el fin de desambiguar el mensaje emitido por el interlocutor. Detectamos esta habilidad a los 3 años cuando el pequeño es capaz de desambiguar una producción si lo cree conveniente con el objeto de asegurar la correcta interacción discursiva. Este comportamiento se encuentra en la línea de los resultados obtenidos por Clark (1978). Los ejemplos siguientes ilustran esta cuestión:

\{Niño de 3 años\}

$$
\begin{array}{ll}
\text { Adulto } & \begin{array}{l}
\text { : y qué más tienes } \\
\text { \{se refiere a los juguetes\} }
\end{array} \\
\text { Niño } & : \text { aquí? }
\end{array}
$$

\{Niño de 4a.6m. El entrevistador pregunta qué hizo en las pasadas vacaciones\}

Adulto : y las vacaciones que pasaron?

Niño : las que pasaron ya?

Niño 5 años\}

Adulto : : dame la caja amarilla

Niño : cual amarilla?

\{Existen diferentes cajas amarillas\}

Reorganización de la información

A los $2 \mathrm{a} .10 \mathrm{~m}$. se han detectado producciones en las que el niño reorganiza la información que está emitiendo para ajustarla a las necesidades del discurso y a sus intenciones comunicativas. En el siguiente ejemplo se observa la reorganización que hace un niño de su propia intención comunicativa:

\{Niño de 3 años. Habla de sus planes de cazar mariposas en sus próximas vacaciones\}

Niño : $\quad$ Si, (un) se ponen a comer en>

\{abandona la oración\} 
Y el tenía que pegar cuando, cuand* ven*>

\{abandona la oración\}

Cuando estaban parados

\section{Referencias a lo dicho por el interlocutor}

A los $2 \mathrm{a} .2 \mathrm{~m}$. se detectan los primeros usos de estilo directo/indirecto que hacen referencia a lo dicho por el interlocutor. A los $5 \mathrm{a} .6 \mathrm{~m}$. se eleva el uso de esta categoría, posteriormente se detecta un descenso hasta los $6 a .6 \mathrm{~m}$. en que se registra nuevamente una mayor frecuencia de uso. Observamos que en estas edades el niño ya tiene un dominio importante de las estructuras sintácticas que le ayudan a reproducir lo dicho por alguien de forma más compleja.

\{Niño de 6a.6m. Emite la producción ante un cuento en imágenes\}

Niño

: Que el (del) castillo le dice ¿tiene un sitio para dormir?

A la edad de 6 años se han detectado alusiones no a lo dicho por alguien, sino a lo que se supone que su interlocutor podrá decir. El niño se anticipa mostrando un conocimiento más profundo de su interlocutor y de su estilo lingüístico.

\{La entrevistadora pregunta qué hará al día siguiente en clase\}

Niño

: (:) no lo sé porque la señu lo tiene que decir

Dirá:

atended chicos

mañana vamos al cine

\section{Adecuación discursiva}

La adecuación discursiva se ha detectado a los $2 \mathrm{a} .2 \mathrm{~m}$. cuando el niño es capaz de reproducir estilos propios de determinados contextos y registros:

\{Niña 2a.2m. La niña y la entrevistadora están jugando y al ver una manzana la niña reproduce la interacción entre un vendedor y su madre\} 
RLA. Revista de Lingüística Teórica y Aplicada, 47 (1), I Sem. 2009

Niña : Toma, eto, toma, toma.

Toma apanta (:3)

¿Quie poma Sole?

\{Poma es una palabra catalana que significa manzana\}

Podemos apreciar que en esta producción la niña imita y dista todavía mucho de mostrar una plena reflexión metalingüística tal y como afirma Gombert (1990). No obstante, la pequeña se apercibe de la existencia de diferentes registros e imita personajes sobre todo cambiando al principio la voz o la entonación.

\section{Referencia al habla o al idioma de los interlocutores}

La referencia al habla del interlocutor se ha detectado a los 22 meses. Se detectaron producciones que hacen referencia a la lengua de los interlocutores como podemos ver a continuación:

$\{$ Niña de 2a.6m.\}

Niña : Mo bé

Mamá Ana no diu mo bé

\{bé significa bien en catalán\}

Diu mu bié

\{la niña intenta decir bien en castellano\}

\{La niña juega con los muñecos, entre ellos había un abuelo y una abuela. Mientras juega con éstos hace referencia a su abuela quien dice muy bien en lugar de molt bé -en catalán-. Diferencia ambos idiomas\}.

$\{$ Niño de 2a.6m. $\}$

Niño : : : yo, yo no, (no) yo anam amb barc

Adulto : ¿¿qué?

Niño : : yo ya sé catalán

\{El niño hace una adaptación del castellano al catalán, de aquí que diga que ya sabe catalán. Barc es una adaptación de barco y la forma verbal anam está mal conjugada. La traducción al castellano es "vamos con barco", la producción correcta en catalán sería anem amb vaixell\}

En el primer ejemplo podemos apreciar la habilidad de la niña para hacer 
referencia a las formas de hablar de dos personas (las identifica y compara). En el segundo ejemplo el niño adapta las estructuras de dos idiomas. Creemos que la precocidad con que se ha detectado esta categoría en nuestro trabajo es atribuible a la situación de bilingüismo en que se encuentran inmersos los pequeños, pero para comprobar este aspecto necesitaríamos profundizar más en el tema.

A los 6a.6m. encontramos casos en los que el niño es capaz de atribuir a un ser inanimado procesos o acciones, en este caso relacionados con un supuesto idioma que habla un león de peluche.

\{Niño de 6a.6m.\}

Niño : no me entiende porque yo no soy un león y no hablo como él.

\section{CONCLUSIONES}

El objetivo que nos ha servido como punto de partida para la realización de este trabajo era el profundizar tanto en las secuencias de desarrollo y estabilización de las habilidades metalingüísticas, como en los diferentes momentos (más o menos oscilantes) de emergencia de estas habilidades. Para poder conseguir esta meta nos hemos apercibido que resulta necesario trabajar con amplias franjas de edad que permitan indicar los logros y dificultades infantiles en el acceso a las habilidades metalingüísticas de regulación de la información.

Los resultados nos han permitido apreciar una secuencia y estabilización de las habilidades metapragmáticas relacionadas con la gestión de la comunicación. Ahora bien, estas habilidades no emergen simultáneamente. En un primer momento aparecen aquéllas encaminadas a que el niño pueda adquirir nueva información, aumentando de esta forma su competencia tanto lingüística como metalingüística. A continuación se detecta otro grupo de categorías de mayor complejidad y que son el reflejo de esta evolución, mostrándose en este punto la retroalimentación entre ambas competencias. Este progreso se hace patente, por ejemplo, en la regulación basada en el interlocutor como centro de interés que aparece de forma más tardía cuando el niño posee un mayor grado de competencia y dispone de estructuras que le permiten utilizar esta categoría, excepto en el caso de las referencias al habla $o$ al idioma de los interlocutores que se ha detectado a una edad más temprana.

El análisis cuantitativo nos ha permitido observar resultados que nos advierten sobre la precocidad de ciertas conductas, por ejemplo la capacidad de detectar si el interlocutor ha comprendido o no el mensaje recibido (Clark, 1978), pero también el análisis cualitativo de las producciones nos muestra que estas habilida- 
des se desarrollan más tarde de manera más sólida y consistente (Gombert, 1990; Markman, 1977, 1981).

Los intercambios analizados también ponen de relieve la importancia de contextualizar la emergencia de las producciones metalingüísticas (Edwards \& Kirkpatrick, 1999; San Martín y Torres, 2004). Esta cuestión nos permite sugerir que un contexto bilingüe parece favorecer cierta toma de conciencia lingüística, pudiendo adelantar las edades de aparición de ciertas producciones; no obstante para poder afirmar este aspecto necesitaremos comparar niños monolingües y bilingües, es decir, niños procedentes de ambos contextos. Por otra parte, también es de gran interés poder profundizar en el papel del interlocutor más experto, en nuestro caso el adulto, dado que éste juega un papel decisivo en el desarrollo de las habilidades metalingüísticas guiando y ayudando al niño en todo momento, mostrando la existencia de un sistema bidireccional. Por último, creemos que resulta de interés profundizar la interacción entre el nivel cognitivo y el lingüístico en la línea de las propuestas de Beaudichon \& Ducroux (1985). Esta interacción se hace evidente en aquellos casos en los que el niño debe ponerse en el lugar de su interlocutor para comprender qué información necesitará e incluso prever lo que pueda decir éste.

Teniendo en cuenta todos los aspectos mencionados hasta el momento, la diversidad de resultados encontrados en la literatura consultada se entiende en relación a las características de los contextos de interacción, las demandas de la tarea, el nivel cognitivo, la experiencia y guía en la misma, etc. En este sentido, creemos que la observación en contextos comunicativos naturales y espontáneos, así como el contraste con otros contextos comunicativos, puede ser de gran utilidad para estudiar estos procesos.

Las derivaciones de este tipo de trabajos son importantes. No debemos olvidar la importancia de la capacidad metalingüística en el contexto escolar, tanto en el caso del aprendizaje de la lengua escrita como en el aprendizaje de segundas lenguas y en la adquisición de las distintas áreas de conocimientos en las que el dominio de las habilidades metalingüísticas y lingüísticas es determinante para comprender sus contenidos. En todas estas áreas el estudio de la evolución de las habilidades metalingüísticas es una herramienta útil en el proceso de enseñanza y aprendizaje. Por otra parte, en el caso de los trastornos del lenguaje el conocimiento de la evolución de las habilidades metalingüísticas relacionadas con la gestión de la comunicación es útil tanto para la detección de una posible alteración en la evolución de estas habilidades como para su intervención.

Para concluir, podemos decir que mediante la función metapragmática y más concretamente mediante la regulación de la información el niño se acomoda y aprende a integrarse en el grupo sociolingüístico al que pertenece. En este sentido, la forma de expresarse de una persona puede marcar positiva o negativamente esta inserción. Si se detecta un problema de índole metapragmático, podríamos estar 
ante la presencia de una patología que supondría para el pequeño un riesgo de desadaptación, ya que el lenguaje y su uso pueden limitar o potenciar diferentes aspectos en la promoción social de una persona.

\section{REFERENCIAS}

Anderson, Elaine. 1992. Speaking with style. The sociolinguistic skills of children. London: Routledge.

Beaudichon, Janine \& Ducroux, Noëlle. 1985. "L'approche du contrôle cognitif de la communication chez l'enfant: Quelques données, quelques problèmes". En Bideaud, J. \& Richelle, M. (eds.), Psychologie dévelopmentale, problèmes et réalités. Bruxelles: Mardaga, pp. 139-159.

Berthoud-Papandropoulou, Ioanna. 2000. "Lo sviluppo della competenza metalinguistica nel bambino", en Metis 1, pp. 7-25.

Clark, E.V. 1978. "Awareness of language: Some evidence from what children say and do". En Sinclair, A., Jarvella, R.J. \& Levelt, W.J.M. (eds.), The child's conception of language. Berlin: Springer, pp. 17-43.

Crystal, David, Fletcher, Paul \& Garman, Michael, M. 1976. The grammatical analysis of language disability: A procedure for assessment and remediation. London: Edward Arnold.

Edwards, Harold \& Kirkpatrick, Anita. 1999. "Metalinguistic awareness in children: A developmental progression", en Journal of Psycholinguistic Research 4 (28), pp. 313-329.

Gombert, Jean Emile. 1990. Le développement métalinguistique. Paris: P.U.F.

Hickman, Maya. 1985. "Metapragmatics in child language". En Mertz, E. \& Parmentier, R.J. (eds.), Semiotic mediation: Sociocultural and psychological perspectives. New York: Academic Press, pp. 177-201.

Karmiloff-Smith, Annette. 1986. "From meta-processes to conscious access: evidence from metalinguistic and repair data", en Cognition 23, pp. 95-147.

Karmiloff, Kyra \& Karmiloff-Smith, Annette. 2001. Pathways to language. From fetus to adolescent. London: Harvard University Press.

Markman, Ellen M. 1977. "Realizing that you don't understand: a preliminary investigation", en Child Development 48, pp. 986-992.

Markman, Ellen M. 1979. "Realizing that you don't understand: Elementary school children's awareness of inconsistencies", en Child Development, 50, pp. 643-55.

Markman, Ellen M. 1981. "Comprehension monitoring". En Dickson, W.P. (ed.), Children's oral communication skills. New York: Academic Press, pp. 61-84.

Miler, Jon \& Chapman, Robin. 1985. Systematic Analysis of Language Transcript (SALT). Madison: University of Wisconsin.

Navarro, $M^{a}$ Isabel. 2007. "Desarrollo de las habilidades metapragmáticas: Estudio longitudinal de un niño con trastorno especifico del lenguaje". Comunicación presentada en el II Congreso Internacional de Aprendizagem na Educaçao de Infancia. Oporto. 
Navarro, Maa Isabel y Hernández, E. 2004. "Desarrollo de las habilidades metalingüisticas: Aplicación a la evaluación e intervención de un niño con un défcit semántico pragmático". Comunicación presentada en el II Congreso Internacional de Atención Temprana. Santiago de Compostela, España.

Navarro, Ma Isabel y San Martín, Conchi. 2007. "Desarrollo de las habilidades metalinguísticas durante la edad escolar". Comunicación presentada en el II Congreso Internacional de Aprendizagem na Educaçao de Infancia. Oporto.

Rondal, J.A., Espert, J.E., Gombert, E., Thibaut, J.P. \& Comblain, A. 2003. "Desarrollo del lenguaje oral”. En Puyuelo, M. y Rondal, J.A. (eds.), Manual de desarrollo y alteraciones del lenguaje. Aspectos evolutivos y patología en el niño y el adulto. Barcelona: Masson, pp. 42-48.

San Martín, Concepción y Torres, Empar. 2004. "Estudio de la emergencia del habla privada en una situación comunicativa infantil de juego y sus relaciones con el conflicto comunicativo", en Anuario de Psicología 35, pp. 71-86.

Tunmer, William, Bowey, Judith \& Grieve, Robert. 1983. "The development of young children's awareness of the word as a unit of spoken language", en Journal of Psycholinguistic Research 12, pp. 567-594. 
Anexo1: Sistema de categorización para el análisis de la Regulación de la comunicación (extraído de Navarro y Hernández, 2004).

El sistema utilizado en este trabajo está dividido en dos grupos: a) Regulación y ajustes de la comunicación y b) Regulación basada en el interlocutor como centro de interés. A continuación se indican las definiciones operativas de las categorías consideradas para cada grupo así como una ejemplificación de las mismas.

a) Regulación y ajustes de la comunicación. En este grupo categorial se contemplan las siguientes cuatro categorías:

1. Dar información de carácter metalingüistico. El niño da información de carácter metalingüístico para establecer una interacción comunicativa con éxito.

\{Niño de 3 años $\}$

Niño : toma acalados

Adulto : qué es eso de acalados?

Niño : bacalao

es pa come.

2. Obtener información de carácter metalingüistico. El niño realiza preguntas explícitas de carácter metalingüístico, con el fin de obtener información y poder mantener la comunicación con éxito.

\{Niña 5 años\}

Niña : quién es ella?

Adulto : ella es la muñeca.

3. Detección de incoherencias en el discurso. Manifiesta verbalmente su desacuerdo con la producción lingüística (incoherencia) del interlocutor mediante expresiones metalingüísticas.

\{Niña de 6 años\}

Adulto : así, Ana fue al parque

Niña : : no esa Ana estaba con una fiesta de cumpleaños

la otra Ana la Anita.

4. Reorganización de la información. La producción del niño no concuerda con sus intenciones comunicativas o detecta algún error y procede a realizar una reorganización de la información.

\{Niño: 6 años 5 meses\}

Niño : en las vacaciones voy al pueblo

porque (yo siempre porque te*) sabes eh? no

hay una piscina cerca de casa que está en el pueblo

y me gusta ir 
RLA. Revista de Lingüística Teórica y Aplicada, 47 (1), I Sem. 2009

b) Regulación basada en el interlocutor como centro de interés. En este grupo categorial se contemplan las siguientes tres categorías:

1. Referencias a lo dicho por el interlocutor. Hace referencia a lo dicho en el discurso por los interlocutores, ya sea de forma directa o indirecta.

\{Niño de 7 años\}

Niño : mira, me ha dicho

no chutes fuerte la pelota

o no te dejaré jugar

2. Adecuación discursiva. Se adapta a las características de su interlocutor, empleando un registro discursivo distinto al habitual.

\{Niña de 7años mientras juega a médicos\}

Niña

: Buenos días señora

Qué le pasa a su hijo?

le duele a su hijo la barriga

Ha comido muchos dulces?

Le voy a recetar un jarabe

Y tú no comas tantos dulces que puedes tener caries.

3. Referencia a las características del habla o idioma de los interlocutores. Expresiones donde se hace referencia expresa a algún aspecto de la manera de hablar de los interlocutores o de su propia lengua.

$\{$ Niño de 6años $6 \mathrm{~m}$.

Niño : mila soy un chino

y hablo como un chino

Niño

: Cuando habla

Parece un lorito, no para

Mira como dice Pepe 